\title{
Effect of Corticosteroids in the Acute Management of Pediatric Orbital Cellulitis With Subperiosteal Abscess
}

\author{
Michael T. Yen, M.D.*, and Kimberly G. Yen, M.D.*† \\ *Cullen Eye Institute, Department of Ophthalmology, Baylor College of Medicine, Houston, Texas; and the $\dagger$ Departments of \\ Ophthalmology and Pediatrics, Texas Children's Hospital, Houston, Texas, U.S.A.
}

\begin{abstract}
Purpose: To determine the effect of intravenous corticosteroids in the acute management of pediatric orbital cellulitis with subperiosteal abscess.

Methods: The inpatient records of all patients treated for orbital cellulitis with subperiosteal orbital abscess between January 2001 and August 2003 were reviewed. The use of corticosteroids, length of hospital stay, need for surgical drainage, treatment course, and clinical outcomes were reviewed. A $t$ test and Fisher exact test analysis were calculated to evaluate statistical significance.

Results: Twelve patients received intravenous corticosteroids and 11 patients did not receive corticosteroids. All patients had complete resolution of their abscess without complications. Length of hospitalization between the patients treated with and without intravenous corticosteroids was not significantly different $(p=0.26)$. Four of 12 patients treated with intravenous corticosteroids underwent orbitotomy for drainage of the abscess, and 6 of 11 patients treated without intravenous corticosteroids underwent surgical drainage $(p=0.20)$. Two of 12 patients treated with corticosteroids received intravenous antibiotics after discharge, whereas 7 of 11 in the group not treated with corticosteroids received intravenous antibiotics after discharge $(p=0.03)$.

Conclusions: The use of intravenous corticosteroids does not appear to adversely affect clinical outcomes and may be beneficial in the treatment of pediatric orbital cellulitis with subperiosteal abscess. Review of our data suggests that a prospective, randomized trial is warranted to further clarify the role of corticosteroids in the acute management of pediatric orbital cellulitis with subperiosteal abscess.
\end{abstract}

$\mathrm{O}$ rbital cellulitis with subperiosteal abscess in the pediatric patient is most frequently caused by secondary extension of infection and/or inflammation from the paranasal sinuses. ${ }^{1,2}$ Untreated, subperiosteal orbital abscesses can lead to vision loss, compressive optic neuropathy, cavernous sinus thrombosis, meningitis, or intracranial abscess formation. The treatment of subperiosteal abscesses in the pediatric age group remains controversial. Broad-spectrum intravenous antibiotics are required in all patients. Surgical drainage may be necessary in patients not improving with antibiotics alone, in patients with impending visual loss, or in patients with concurrent cavernous sinus or intracranial involvement. ${ }^{3-6}$

Because successful management of the adjacent sinusitis is paramount in facilitating the resolution of the subperios-

\footnotetext{
Accepted for publication April 11, 2005.

Supported in part by an unrestricted grant from Research to Prevent Blindness, Inc., New York, New York, U.S.A.

Presented in part at the American Society of Ophthalmic Plastic and Reconstructive Surgery Fall Scientific Symposium, New Orleans, Louisiana, October 2004.

The authors have no financial interest in any of the techniques or materials described herein.

Address correspondence and reprint requests to Dr. Michael T. Yen, Cullen Eye Institute, 6565 Fannin, NC-205, Houston, TX 77030, U.S.A. E-mail: myen@bcm.tmc.edu
}

DOI: 10.1097/01.iop.0000179973.44003.f7 teal abscess, any therapy that aids the treatment of the sinusitis may also have a beneficial effect in the treatment of the subperiosteal abscess. A number of studies in the medical literature have reported increased levels of a variety of inflammatory cytokines in the sinonasal mucosa of patients with sinusitis. $^{7-11}$ Treatment with intravenous corticosteroids has been shown to decrease levels of some of these inflammatory cytokines and therefore facilitate the treatment of acute and chronic sinusitis. ${ }^{12-14}$ Intravenous steroids have also been shown to decrease mucosal edema, potentially helping with sinus drainage. ${ }^{15}$ Because intravenous corticosteroids are often used in the management of acute sinusitis at our institution, we postulated that corticosteroids may also have a beneficial role in the management of pediatric subperiosteal abscesses.

\section{METHODS}

Institutional review board approval was obtained for this retrospective review. The inpatient records of all patients treated for orbital cellulitis with subperiosteal orbital abscess between January 2001 and August 2003 at the Texas Children's Hospital were identified by using ICD-9 diagnostic codes. Patients with orbital cellulitis not demonstrating an identifiable subperiosteal abscess on CT or MRI were excluded from the study. Patients who received intravenous corticosteroids during their admission but not as part of the initial management of the subperiosteal abscess were 


\section{Length of Hospitalization}

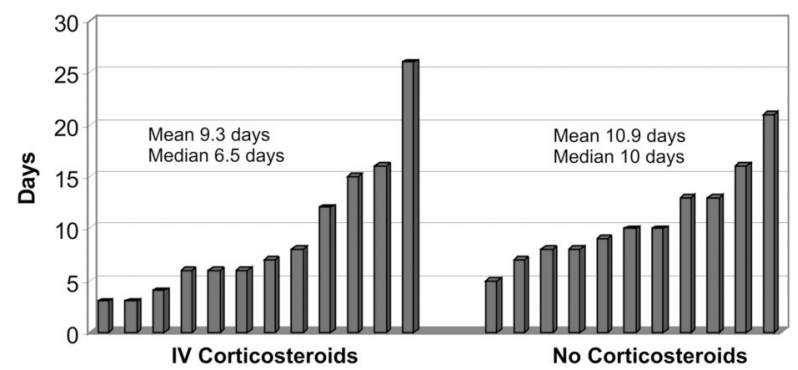

FIG. 1. Median length of hospitalization was reduced in patients receiving intravenous corticosteroids.

also excluded from the study. The use of corticosteroids, length of hospital stay, need for surgical drainage, treatment course, and clinical outcomes were reviewed. A $t$ test and Fisher exact test analysis were calculated to evaluate statistical significance.

\section{RESULTS}

A total of 24 patients were identified, ranging in age from 11 days to 15 years. One patient was excluded because the corticosteroids were not given in the acute treatment of the orbital cellulitis. Twelve patients received intravenous corticosteroids and 11 patients did not receive corticosteroids during their hospitalizations (Tables 1 and 2, available online at www. op-rs.com). Treatment with the corticosteroids began at the time of admission in all 12 patients. Length of hospitalization between the patients treated with and without intravenous corticosteroids was not significantly different $(p=0.26)$, although there was a slight trend toward shorter hospitalizations in the patients treated with intravenous corticosteroids (median, 6.5 days versus 10 days) (Fig. 1). Four of the 12 patients treated with intravenous corticosteroids underwent orbitotomy for drainage of the abscess, and 6 of the 11 patients treated without intravenous corticosteroids underwent surgical drainage $(p=0.20)$. Two of the 12 patients treated with corticosteroids received intravenous antibiotics after discharge, whereas 7 of the 11 in the group not treated with corticosteroids received intravenous antibiotics after discharge $(p=0.03)$ (Fig. 2 ). All patients in both groups had complete resolution of their subperiosteal abscess. Fig. 3

\section{DISCUSSION}

The most frequent cause of pediatric orbital subperiosteal abscesses is acute sinusitis, especially that involving the ethmoid and maxillary sinuses. There is currently no definitive standard for the treatment of subperiosteal abscesses in the pediatric age group. Some argue that an initial trial of intravenous antibiotics is warranted, reserving surgical treatment for patients who do not respond to antibiotic therapy, whereas others believe that immediate surgical drainage of the abscess and infected sinuses is required. ${ }^{2-6,17-20}$ Regardless of whether surgical intervention is pursued or not, the treatment of the concurrent sinusitis is integral to the management of orbital subperiosteal abscesses. Although broad-spec-

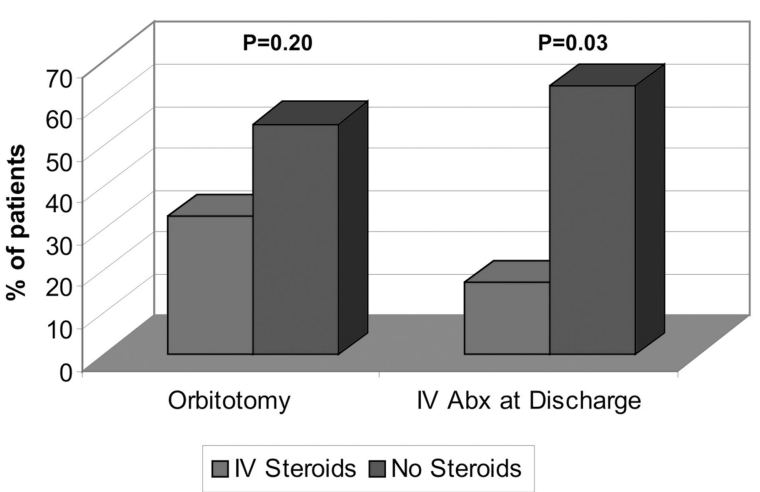

FIG. 2. Fewer patients required surgical drainage of the orbital abscess and intravenous antibiotics at discharge when treated with intravenous corticosteroids.

trum antibiotics are always used in the initial management of the subperiosteal abscess, the use of corticosteroids to treat the orbital subperiosteal abscess has not been well investigated.

At our institution, many otolaryngologists frequently use intravenous corticosteroids as an adjunct therapy in the treatment of acute and chronic sinusitis in addition to antibiotics, nasal decongestants, and nasal saline irrigations. Elevated levels of various proinflammatory cytokines have been found in the sinonasal mucosa of patients with sinusitis, and, after treatment with corticosteroids, mucosal biopsy specimens have demonstrated reduction in the levels of some of these cytokines. ${ }^{7}$ It is believed that the inflammatory response of sinusitis is associated with elevated levels of these cytokines and that medically recalcitrant sinusitis may be associated with a prolonged state of inflammation rather than a persistent bacterial infection. Corticosteroid use after endoscopic sinus surgery in children has also been shown to result in less mucosal edema, scarring, septae formation, and lower incidence of closure of the maxillary ostium. ${ }^{16}$

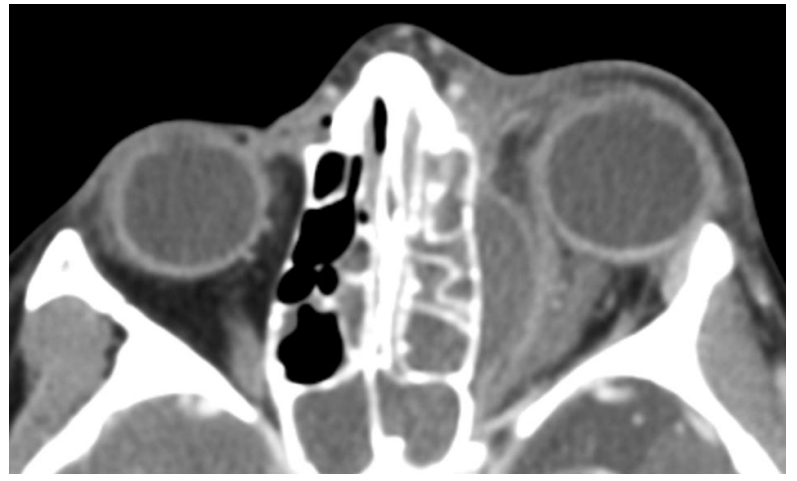

FIG. 3. CT scan of a 6-year-old boy with a large posterior medial orbital subperiosteal abscess. The patient was treated with a single dose of intravenous dexamethasone and broadspectrum intravenous antibiotics for 5 days. He was discharged home on the sixth day after admission on oral antibiotics. 
Although the origin of orbital cellulitis with subperiosteal abscesses is clearly infectious, the presence and size of the abscess does not necessarily correlate directly with the severity of the orbital infection. Many authors have reported "sterile" abscesses despite prompt surgical drainage before the effects of intravenous antibiotics would be anticipated. ${ }^{19,21}$ This suggests that rather than being an aggressive, uncontrolled infection of orbital tissues, the orbital abscess may, in some cases, be due in part to an exuberant inflammatory response to the underlying sinusitis that has spilled over into the subperiosteal space. Controlling and suppressing this exuberant inflammatory response may be a potential benefit from intravenous corticosteroids used in conjunction with broad-spectrum antibiotics. Furthermore, any treatment that facilitates the resolution of the sinusitis should also be beneficial in the treatment of orbital cellulitis with the subperiosteal abscess.

In our study, initial treatment with corticosteroids clearly did not adversely affect clinical outcomes. No patients had adverse reactions to the use of intravenous corticosteroids, and all patients had resolution of the subperiosteal abscesses after treatment. The difference in length of hospitalization and need for surgery was not significantly different between the patients treated with corticosteroids and those treated without corticosteroids. Furthermore, a statistically significant smaller number of patients required intravenous antibiotic use after discharge when treated with corticosteroids. Although these results may not show significant advantages in using intravenous corticosteroids, they strongly suggest that intravenous corticosteroids do not adversely affect outcomes.

Although our initial goal in reviewing the records of those patients receiving corticosteroids for treatment of their sinusitis was to see if there might have been improved clinical outcomes with regard to their subperiosteal abscess as well, without a prospective, randomized trial, the exact role of corticosteroids is not certain. Due to the many limitations of our current review, we cannot confidently conclude that the use of intravenous corticosteroids actually improves clinical outcomes. Although there are many theoretical advantages for the adjunct use of intravenous corticosteroids, ${ }^{7-11,13-16}$ our review was not adequate in determining a true link between corticosteroids and improvement. For example, even though our analysis showed that significantly fewer patients required intravenous antibiotics after discharge when treated with intravenous corticosteroids, we cannot determine through our review which clinical parameters were used in deciding whether or not intravenous antibiotics were needed. This further emphasizes the need for a prospective, randomized trial to clarify the role of corticosteroids in the acute management of pediatric orbital cellulitis with subperiosteal abscess.

Major limitations of retrospective reviews include lack of standardization for treatment, potential selection bias, and lack of control of other potentially influential factors. For example, all patients reviewed had different consultants and surgeons with different criteria for surgery and antibiotic treatment. This chart review was unable to determine if management decisions were based on specific clinical parameters or physician/surgeon practice patterns. In some cases, despite clear documented clinical improvement, patients who received intravenous corticosteroids remained in the hospital for no apparent reason other than the customary protocol for a particular physician. This may explain why the mean length of hospitalization and need for surgical drainage seemed to approach but did not quite meet statistical significance, yet the median length of hospitalization was much shorter for patients treated with corticosteroids. There also was no standard dosage for the corticosteroids given, with the patients reviewed in this study receiving intravenous dexamethasone ranging from 0.33 to $1 \mathrm{mg} / \mathrm{kg}$ from a single dose to every 12 hours for 7 days. Additionally, selection bias could potentially influence the results of our review, since the majority of the patients treated with corticosteroids had medial orbital subperiosteal abscesses, which are more likely to resolve without surgical intervention even without intravenous corticosteroids.

In summary, although the optimum dose and timing has not been determined, the use of intravenous corticosteroids does not appear to adversely affect clinical outcomes and may be beneficial in the treatment of pediatric orbital cellulitis with subperiosteal abscess. In our review, patients treated with intravenous corticosteroids were much less likely to require intravenous antibiotic treatment at discharge. Review of our data suggests that a prospective, randomized trial is warranted to further clarify the role of corticosteroids in the acute management of pediatric orbital cellulitis with subperiosteal abscess.

\section{REFERENCES}

1. Sobol SE, Marchand J, Tewfik TL, et al. Orbital complications of sinusitis in children. J Otolaryngol 2002;31:131-6.

2. Rahbar R, Robson CD, Petersen RA, et al. Management of orbital subperiosteal abscess in children. Arch Otolaryngol Head Neck Surg 2001;127:281-6.

3. Garcia GH, Harris GJ. Criteria for nonsurgical management of subperiosteal abscess of the orbit: analysis of outcomes $1988-$ 1998. Ophthalmology 2000;107:1454-6.

4. Greenberg MF, Pollard ZF. Medical treatment of pediatric subperiosteal orbital abscess secondary to sinusitis. J AAPOS 1998;2:351-5.

5. Sajjadian A, Chundru U, Isaacson G. Prospective application of a protocol for selective nonsurgical management of suspected orbital subperiosteal abscesses in children. Ann Otol Rhinol Laryngol 1999;108:459-62.

6. Rubin SE, Rubin LG, Zito J, et al. Medical management of orbital subperiosteal abscess in children. J Pediatr Ophthalmol Strabismus 1989;26:21-7.

7. Lennard CM, Mann EA, Sun LL, et al. Interleukin-1 beta, interleukin-5, interleukin-6, interleukin-8, and tumor necrosis factoralpha in chronic sinusitis: response to systemic corticosteroids. Am J Rhinol 2000;14:367-73.

8. Kassim SK, Elbeigermy M, Nasr GF, et al. The role of interleukin- 
12, and tissue antioxidants in chronic sinusitis. Clin Biochem 2002;35:369-75

9. Repka-Ramirez S, Naranch K, Park YJ, et al. Cytokines in nasal lavage fluids from acute sinusitis, allergic rhinitis, and chronic fatigue syndrome subjects. Allergy Asthma Proc 2002;23:185-90.

10. Rudack C, Stoll W, Bachert C. Cytokines in nasal polyposis, acute and chronic sinusitis. Am J Rhinol 1998;12:383-8.

11. Bachert C, Wagenmann M, Rudack C, et al The role of cytokines in infectious sinusitis and nasal polyposis: Allergy 1998;53:2-13.

12. Rubin SE, Zito J. Orbital subperiosteal abscess responding to medical therapy. J Pediatr Ophthalmol Strabismus 1994;31:325-6.

13. Cable BB, Wassmuth Z, Mann EA, et al. The effect of corticosteroids in the treatment of experimental sinusitis. Am J Rhinol 2000;14:217-22.

14. Wallwork B, Coman W, Feron F, et al. Clarithromycin and prednisolone inhibit cytokine production in chronic rhinosinusitis. Laryngoscope 2002;112:1827-30.

15. Gehanno P, Beauveillain C, Bobin S, et al. Short therapy with amoxicillin-clavulanate and corticosteroids in acute sinusitis: results of a multicentre study in adults. Scand J Infect Dis 2000;32:679-84.

16. Ramadan HH. Corticosteroids therapy during endoscopic sinus surgery in children: is there a need for a second look? Arch Otolaryngol Head Neck Surg 2001;127:188-92.

17. Greenberg MF, Pollard ZF. Nonsurgical management of subperiosteal abscess of the orbit. Ophthalmology 2001;108:1167-68

18. Harris GJ. Subperiosteal abscess of the orbit: older children and adults require aggressive treatment. Ophthal Plast Reconstr Surg 2001; 17:395-7.

19. Noel LP, Clarke WN, MacDonald N. Clinical management of orbital cellulitis in children. Can J Ophthlamol 1990;25:11-6.

20. Ikeda K, Oshima T, Suzuki H, et al. Surgical treatment of subperiosteal abscess of the orbit: Sendai's ten-year experience. Auris Nasus Larynx 2003;30:259-62.

21. Harris GJ. Subperiosteal abscess of the orbit: Age as a factor in the bacteriology and response to treatment. Ophthalmology 1994;101: 585-95.

\section{COMMENTARY ON EFFECT OF CORTICOSTEROIDS IN THE ACUTE MANAGEMENT OF PEDIATRIC ORBITAL CELLULITIS WITH SUBPERIOSTEAL ABSCESS}

Drs. Michael and Kimberly Yen provide a service in reporting their retrospective review of patients with subperiosteal abscess (SPA) of the orbit treated with intravenous corticosteroids. SPA abscess of the orbit generally arises from the extension of acute ethmoid or maxillary sinusitis. Corticosteroid therapy lessens inflammatory mediators in the sinus mucosa, with presumed subsequent decreases in mucosal congestion and postsurgical scarring. ${ }^{1}$ Corticosteroid therapy is a standard part of the treatment of allergic fungal sinusitis. Inhaled intranasal steroids are shown in multiple randomized studies to increase the efficacy of antibiotic therapy for acute sinusitis. ${ }^{2}$ Nonetheless, concerns arise that systemic corticosteroid use might depress the patient's immune response and allow or mask the progression of infection.

The current study has a number of limitations including the nonrandomized retrospective nature of the data. Numer- ous physicians on several clinical services treated the patients without a standard protocol. There is significant potential in such a series for treatment biases that create non-comparable treatment groups. Furthermore, many of the measured endpoints such as the number of days of hospitalization, days of antibiotic therapy and the use of intravenous versus oral antibiotics are clinically determined without a standardized, objective clinical endpoint or decision tree.

Harris and coauthors have described the indications for medical therapy in orbital SPA as no visual loss, age less than nine, medial location, and no intracranial or frontal sinus involvement. ${ }^{3}$ Review of Yen and Yen's data shows that the abscess was in the favorable medial location in 11 of 12 patients in the steroid treated group, but only 6 of 11 patients in the no steroid group, a trend that was not statistically significant (Fisher exact test). The outcome of subgroups within the two study arms appear similar, as the only non-medial abscess treated with steroids was drained, and 3 of 5 non-medial abscesses in the no steroid group were drained. Of patients age 9 or older, 2 of 4 in the steroid-treated group and 3 of 4 in the no-steroid group required drainage. No entry difference between groups attained statistical significance in this study with relatively small numbers. A suggestion of differences between the study groups raises concern, however, about possible entry bias in the steroid treatment group.

Care must be taken to avoid giving excess significance to the finding of a decreased rate of intravenous antibiotic therapy after discharge in the corticosteroid treated group. The conclusion that corticosteroids sped the patients' clinical resolution is logical within the context of the paper. Alternate interpretations include a decrease in orbital edema in the corticosteroid-treated group not directly associated with resolution of the orbital cellulitis, the possible entry differences discussed previously with noncomparable treatment groups, or a selection bias on the part of the clinicians.

The study by Yen and Yen attracts considerable interest as several institutions and clinical services have used systemic corticosteroids in the treatment of severe acute sinusitis and orbital cellulitis with SPA. This has gone on despite a lack of formal reporting of the results of such treatment. Prospective studies point to the efficacy of intranasal inhaled steroids in speeding the resolution of acute sinusitis ${ }^{2}$ It is reasonable to add inhaled intranasal corticosteroids in cases of orbital subperiosteal abscess being treated medically. It may be reasonable to cautiously consider the addition of systemic steroid therapy in similar cases of subperiosteal abscess in which the

DOI: 10.1097/01.iop.0000180752.57062.36 
initial management is medical therapy only. We hope a prospective, randomized study is forthcoming to appropriately guide therapy.

John B. Holds, M.D.

Departments of Ophthalmology and Otolaryngology-Head and Neck Surgery

Saint Louis University

St. Louis, Missouri

\section{REFERENCES}

1. Ramadan HH. Corticosteroids therapy during endoscopic sinus surgery in children: is there a need for a second look? Arch Otolaryngol Head Neck Surg 2001;127:188-2.

2. Lolor RJ, Witsell DI, Hellkamp AS, et al. Comparison of cefuroxime with or without intranasal fluticasone for the treatment of rhinosinusitis. The FAFFS trial: a randomized controlled trial. JAMA 2001;286:3097-105.

3. Garcia GH, Harris GJ. Criteria for nonsurgical management of subperiosteal abscess of the orbit: analysis of outcomes 1988-1998. Ophthalmology 2000;107:1454-6. 